\title{
Validation of a novel technique that minimises early neonatal deaths -a comparative Study
}

\begin{abstract}
Nigeria did not achieve the millennium development goal (MDG) for the reduction of under-5 mortality (U5M) in 2015. Neonatal death still accounts for nearly $50 \%$ of U5M, hence responsible for this failure. The World Health Organisation records showed that $79 \%$ of deceased neonates would die within the first-seven-days (F7D) of life in Nigeria. Prolonged postnatal delay before attainment of normal body temperature could be partly responsible. Reducing F7D deaths could improve Nigeria's overall record; hence the urgent need to device a technique that could be applied to achieve this. We developed a neonatal temperature control protocol "the initial-setpoint-algorithm (ISA)" as a response to temperature-related high F7D mortality rate. A total 134 cases (105 controls and 29 tests) were recruited. The cases drew from extremely- low birth weight and preterm neonates (BW $=600-1200 \mathrm{~g}, \mathrm{GA}=26-32$ weeks) presenting at the University of Abuja Teaching Hospital Nigeria. Mortality outcomes were compared to investigate how the ISA group performed relative to the Control group. Applying ISA, Test-patients attained normotherm in $0.47 \mathrm{hr}$ post-presenting $(95 \% \mathrm{CI}: 0.2)$; Control took $12.4 \mathrm{hrs}(95 \% \mathrm{CI}: 2.3)$. The F7Ddeaths contribution to mortality in the control group was $71 \%$ (41 out of 58 ). There was no F7D death amongst the only two deceased neonates in the test group. The ISA helped quick attainment of normotherm in the Test-group; hence removed the immediate postnatal long exposure to damaging thermal shock that trailed other neonates to death within the F7D period.
\end{abstract}

Keywords: neonates, first seven days of life, mortality, normotherm, temperature control, neonatal impact
Volume 6 Issue I - 2017

\author{
Amadi HO, ${ }^{1,2,3}$ Adesina CT,' Olateju EK,' \\ Omokaro S,' Okechukwu AA,' Alabi P,' \\ Haruna AS' \\ 'Paediatrics Department, University of Abuja Teaching Hospital, \\ Nigeria \\ ${ }^{2}$ College of Medicine, Imo State University Owerri, Nigeria \\ Bioengineering Department, Imperial College London, UK
}

Correspondence: Professor Hippolite O Amadi, Bioengineering Department, Imperial College London, South Kensington Campus, SW7 2AZ, United Kingdom, Email h.amadi@imperial.ac.uk

Received: September 13, 2016 | Published: January I8, 2017
Abbreviations: MDG, millennium development goal; U5M, under-5 mortality; F7D, first-seven-days of life; NNMR, neonatal mortality rate; F7DMR, mortality rate within the first-seven-days of life; ISA, initial setpoint algorithm; BW, birth weight; GA, gestational age; CI, confidence interval; SCBU, special care baby unit; ELBW, Extremely Low Birth Weight; WHO, World Health Organisation; UATH, University of Abuja Teaching Hospital; WUSP, warm up setpoint; VLBW, very low birth weight; LBW, low birth weight; POA, point of admission; T1, air temperature of neonate's immediate environment; $\mathrm{T} 2$, air temperature of neonate's prospective environment; $\mathrm{Tb}$, neonate's body temperature measured via the axilla; $\mathrm{Td}$, neonate's temperature deviation from the midpoint $\left(36.9^{\circ} \mathrm{C}\right)$ of the physiologic temperature Safe-Zone $\left(36.5^{\circ} \mathrm{C}-37.4^{\circ} \mathrm{C}\right)$; Ti, optimized initial setpoint value; RD, respiratory distress; HyperB, hyperbilirubinemia; HypoT, hypothermia; HyperT, hyperthermia; HypoG, hypoglycemia; HypoN, hypernatraemia; HyperN, hyponatraemia; HyperK, hyperkalaemia; HypoK, hypokalaemia; HypoC, hypocalcaemia

\section{Introduction}

New born babies during the first few days of life often require assistance in the provision of appropriate warmth needed for them to maintain the physiological body temperature of $36.5^{\circ} \mathrm{C}-37.4^{\circ} \mathrm{C}$ for survival. This is referred to as the neonatal thermal safe-zone. ${ }^{1}$ Neonates have high incidence of mortality when exposed outside this temperature range for a long period of time. ${ }^{2}$ The risk of death due to improper neonatal body temperature within few days after birth can increase by over $70 \%$ in extreme prematurity and as birth weight decreases far below $1000 \mathrm{~g} .{ }^{3,4}$ The admission rate of premature low birth weight neonates in most Nigerian special care baby units (SCBU) easily constitutes over $45 \%$ of total admission at any given time. ${ }^{5}$ Insufficient functional knowledge of the dynamics of neonatal thermoneutral control - even with incubators in many Nigerian centres - makes it difficult to achieve a safe-zone body temperature as quickly as it is necessary for the survival of the neonate. Extremely low birth weight neonates (ELBW, $<=1000 \mathrm{~g}$ ) had thus been allowed to remain outside the thermal safe-zone continuously for up to 45 hours prior to death as carers struggled without success in the attempt to thermally stabilize them within the safe-zone temperatures [1]. A study that examined the neonatal spectrum of mortality at four facilities spread across Nigerian regions - between April 2009 and March 2011 revealed that more than five in 10 neonates that died in some centres within 48 hours of presenting were categorized as ELBW. ${ }^{6}$ In another cohort study involving 98 neonates (birth weight $<=1000 \mathrm{~g}$ and or gestation age $<33$ weeks) that died within 7 days of presenting at three Nigerian facilities that were selected from three different regions of the country, complications of improper body temperatures was found to be associated with $83 \%$ of these ${ }^{-1}$ Many of the deceased remained thermally outside the safe-zone temperatures for the entire period of their short lifetime. The strong association of temperature and early neonatal death might be primarily responsible for Nigeria's high neonatal mortality record - as the World Health Organisation (WHO) published that $79 \%$ of deceased Nigerian neonates died within their first seven days (F7D) of life. ${ }^{7}$

There is no recorded evidence of any standard algorithm (defined as a set of guiding rules) - within the Nigerian practice - for determining patient-specific setpoint that could be used to initiate effective incubator intervention for a newly admitted neonate. We hypothesize that the introduction of an effective algorithm would eliminate the observed long periods it takes Nigerian practitioners before they could successfully stabilise a neonate within the thermal safe-zone temperatures and hence minimize mortality. This prolonged abnormal thermal condition immediately following birth seems to 
impact a lasting damage, weakening many of the neonates in their battle to survive the F7D period. The Handy-approach temperature management technique was devised by Amadi (2012) and has been shown to be associated with remarkable improvement of outcome in the SCBU. ${ }^{1,8}$ This however could not eliminate initial thermal devastation often caused by the long periods of early thermal distress experienced by the neonates as the attendants attempt to thermally stabilize the newborn upon arrival. Hence, the aim of this study was to validate a developed "initial-setpoint-algorithm (ISA)" and comparatively investigate its impact on neonatal mortality and morbidity at a Nigerian referral hospital centre. Ethical clearance for this study was obtained from the Research Ethics Committee of the University of Abuja Teaching Hospital (UATH), Gwagwalada Nigeria.

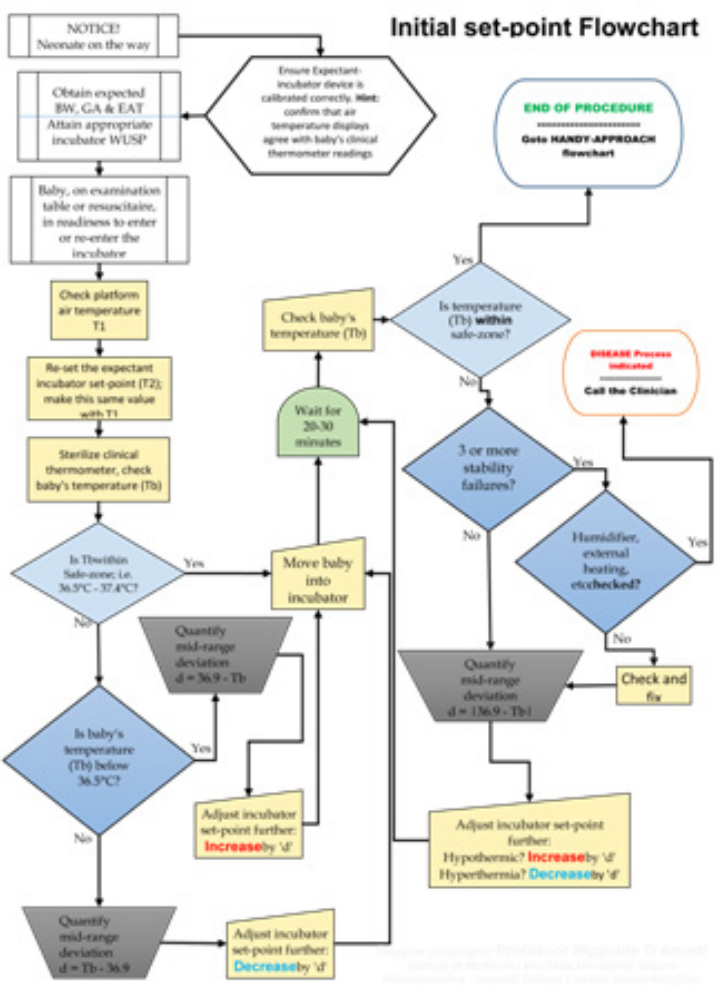

Figure I The initial setpoint algorithm flowchart.

\section{Methods}

A standardized technique was developed as a guide for the quantification of neonate-specific initial temperature set-point of an incubator that could enable a quick attainment of normotherm for the neonate. This was referred to as "the initial setpoint algorithm (ISA)" (Figure 1). The ISA procedures were initiated as soon as the information or notice of 'arriving neonate' was received at the SCBU. This was also initiated when a neonate was to be moved from another environment such as returning to the incubator from an open radiant warmer or open resuscitaire or open cot. We developed the technique based on an earlier successful idea of the "Handy-approach" from the literature. ${ }^{8}$ The technique was used to tackle the lack of algorithm that often manifested in a widespread exposure of a neonate to prolonged thermal distress following a change of environment - as a result of uneducated or arbitrary choice of set-point for the 'new' environment. The technique functioned to provide an educated guess for the patient-specific set-point that would guarantee the neonate's earliest attainment of body temperature within the physiologic thermal safezone.

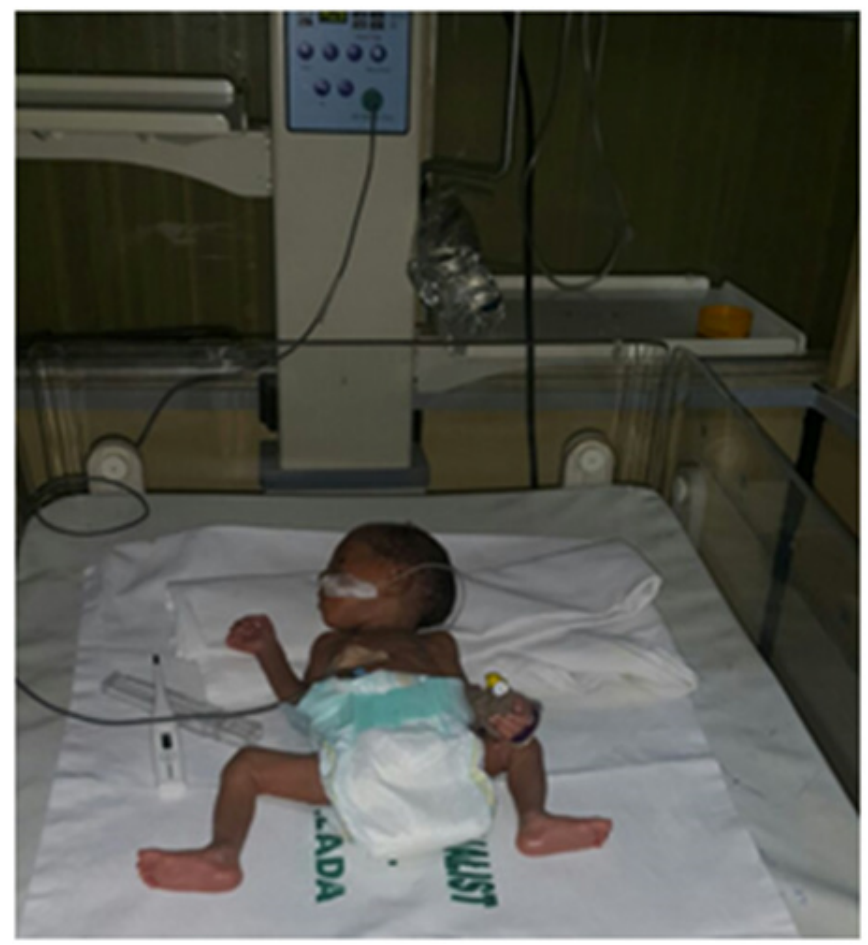

Figure 2 ISA transfer procedure. Air temperature within baby's present immediate environment was measured for the prediction of the patient-specific set-point that would ensure nornotherm in the prospective environment. Thermometer was inclined such that its measuring tip suspended in air without touching any material.
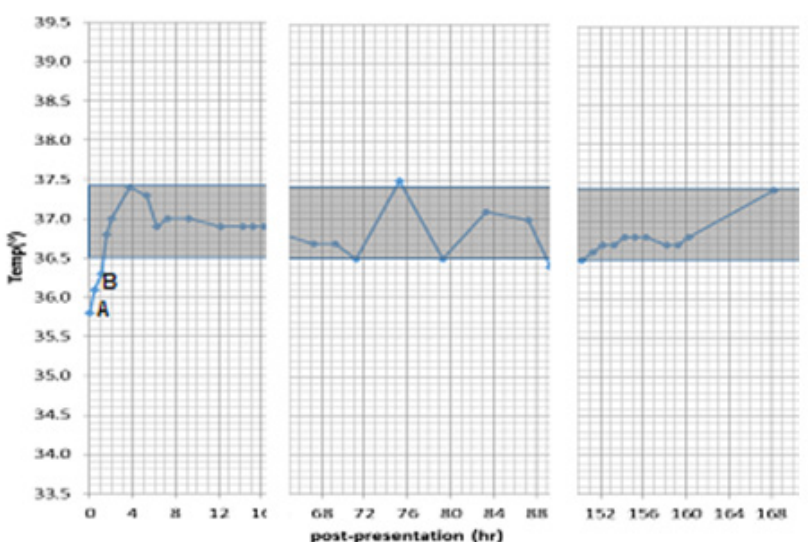

Figure 3 Temperature-Lifetime graph for one of the test-patients (A point of admission, B - commencement of ISA intervention; Greyish band - physiological thermal safe-zone).

\section{Initial-setpoint-algorithm}

The ISA would require the attending clinician or nurse to (1) prepare the 'expectant' thermo neutral system (e.g. the incubator) prior to neonate's arrival whenever possible, (2) assess the neonate's situation upon arrival, and (3) assess the temperature conditions of its current and intending environments as nursing care commenced.

\section{Expectant incubator system}

The incubator system within which the arriving neonate would be nursed was cleaned and powered ' $\mathrm{ON}$ ' - up to 45 minutes prior to 
neonate's arrival to enable adequate time for the incubator to warmup. The warm-up setpoint (WUSP) for the incubator was guided by two vital pieces of information that the ambulatory unit or the Labourward must provide. These were the gestation age (GA) and the birth weight (BW) if any of these was known; else guided estimates were made for these data. The WUSP guided a choice of warm-up temperature that would keep the incubator environment very close to the expected environmental temperature that would be required to normalize the body temperature of a neonate for the given BW or GA. This was determined as follows:

I. An extremely low birth weight (ELBW, $<=1000 \mathrm{~g}$ ) neonate might be completely unable to make autonomous contribution towards generation of body heat and would hence be completely dependent on the absolute environmental temperature [8]. Therefore WUSP for such cases was set at the threshold of hypothermic temperature, i.e. $36.5^{\circ} \mathrm{C}$.

II. A very preterm or very low birth weight (VLBW, 1001g-1500g) neonate might minimally make some autonomous contribution towards thermal stability. Hence, WUSP for this category was set at $36^{\circ} \mathrm{C}$.

III. Low birth weight neonates (LBW, 1501g-2500g) might require a relatively less amount of thermal support, hence WUSP for this category was set at $35.5^{\circ} \mathrm{C}$

IV. For all other bigger neonates that would require incubator support, WUSP was set at $35^{\circ} \mathrm{C}$. Incubator was left to warm up to attain to the set WUSP whilst neonate was awaited or being examined upon arrival.

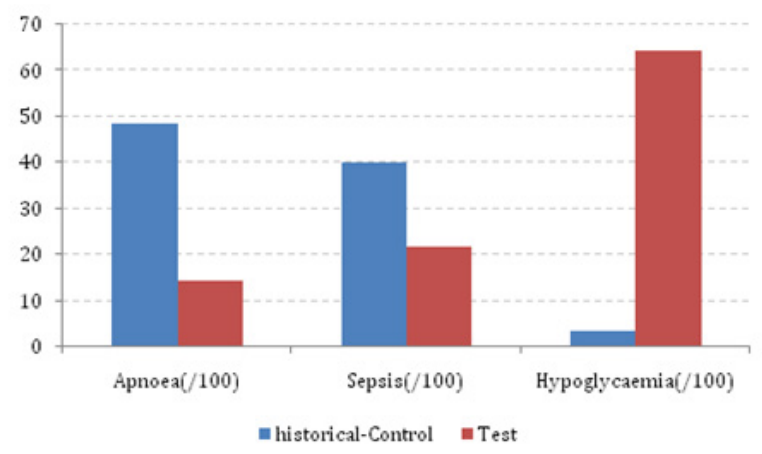

Figure 4 Comparisons of Apnoea, septicaemia and hypoglycaemia.

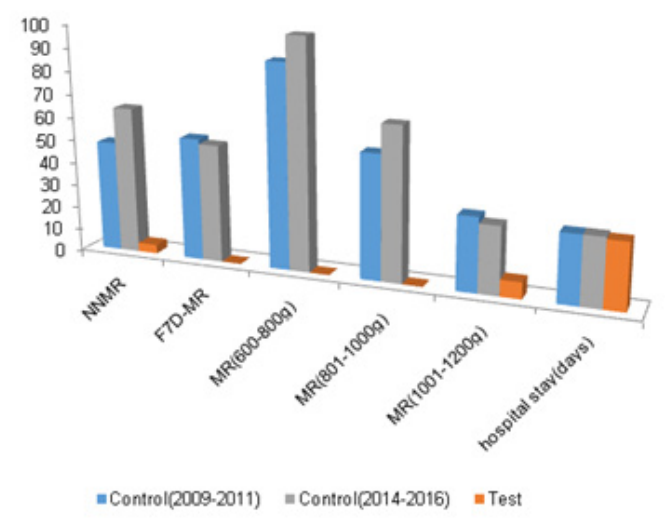

Figure 5 Mortality rates of sub-groups and average hospital stay for survivors (Key: NNMR - neonatal mortality rate, F7DMR - mortality rate within the first-seven-days of life, MR - mortality rate).

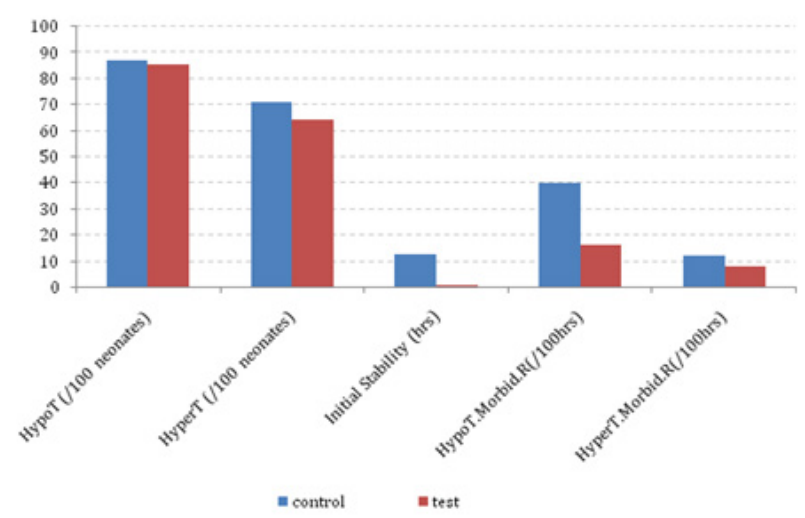

Figure 6 Comparative morbidity due to Hypothermia and hyperthermia (Abbreviations: 'HypoT' or 'HyperT' - hypothermia or hyperthermia presenting within given neonate population, 'HypoT.Morbid.R' - hypothermia morbidity rate defined in terms of the average duration of hypothermia for every 100 hours of nursing).

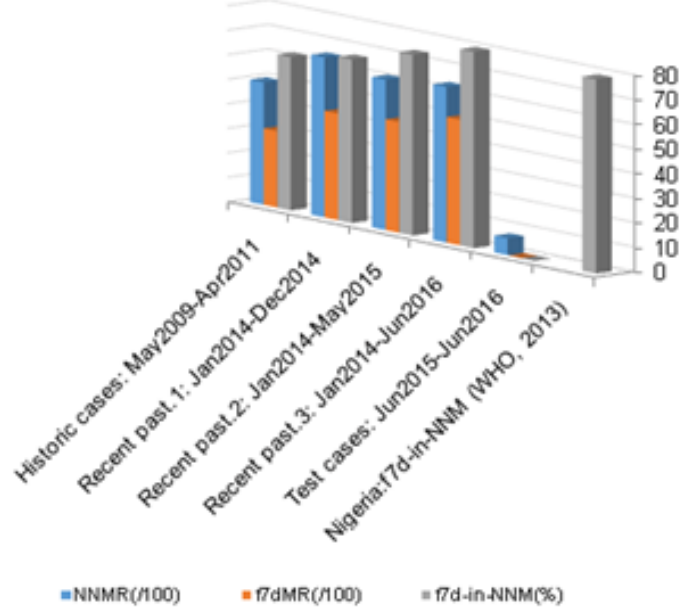

Figure 7 Comparative representation of mortality during the first seven days of life. (NNMR - overall neonatal mortality rate, $f \mathrm{dMR}$ - mortality rate within the F7D period).

\section{Neonate's transfer to expectant incubator}

The adjustment procedure for determining the final initial incubator setpoint and the transfer of the neonate to the expectant incubator system after examination at the point-of-admission (POA) was carried out as follows:

1. Hands were properly washed and new pair of gloves put on.

2. The air temperature of baby's present environment (T1) was measured with a digital thermometer and noted. This environment could be the air at the resuscitaire platform upon which neonate was being examined. Measurement of T1 proceeded as follows:

a. The jacket of baby's digital thermometer was wiped clean with cotton wool wetted in methylated spirit and placed on the mattress beside baby, at its chest level.

b. The thermometer was inclined at an angle, leaning on the jacket such that its measuring tip suspended in the air at the level of neonate's body and a distance of up to $15 \mathrm{~cm}$ from it (Figure 2). 
c. Thermometer was turned ' $\mathrm{ON}$ ' to measure but bearing in mind that most of the available digital thermometers could only measure between $32^{\circ} \mathrm{C}$ to $42^{\circ} \mathrm{C}$. For the occasional environmental temperatures outside this range, alternative thermometer that measured a wider range was used.

3. Set temperature (T2) of neonate's prospective environment (or the expectant incubator) was then changed from its present value (i.e. WUSP) to the same value as T1. This step assumed that if T1 and T2 were the same then neonate was likely going to retain its present body temperature within the same approximate measure of humidification.

4. Baby's digital clinical thermometer was wiped with cotton-woo wetted in methylated spirit for sterilization and used to measure neonate's body temperature $(\mathrm{Tb})$ whilst still on the examination platform.

5. Neonate could now be transferred without any further readjustment of the expectant incubator's or warming-device's setpoint if $\mathrm{Tb}$ was within the thermal safe-zone temperatures.

6. If neonate's temperature was outside the safe-zone then the predicted $\mathrm{T} 2$ required to stabilize neonate in the prospective environment was quantified in two further steps before neonate's transfer thus:

a. The midpoint of the thermal safe-zone - in between the lower $\left(36.5^{\circ} \mathrm{C}\right)$ and upper $\left(37.4^{\circ} \mathrm{C}\right)$ limits - was fixed at $36.9^{\circ} \mathrm{C}$ and used to quantify neonate's thermal deviation (Td) from the midpoint of safe-zone as: $(\mathrm{Td}=|36.9-\mathrm{Tb}|)$

b. The optimal initial set-point value (Ti) was hence quantified as $\mathrm{Ti}=\mathrm{T} 2 \pm \mathrm{Td}$, (i.e. $\mathrm{T} 2$ was further increased by $\mathrm{Td}$ if neonate was found hypothermic or decreased by same amount for hyperthermia).

7. eonate was then transferred and allowed for up to 15 minutes before reassessment to confirm stability within thermal safe-zone.

Subsequent management of the setpoint to ensure that neonate remained within the safe-zone was dynamically maintained by the application of the handy-approach 'flowchart' as described in the literature. ${ }^{8}$ However, for extremely hypothermic neonates with temperatures below $33^{\circ} \mathrm{C}$, caution was taken to ensure that rate of rewarming did not go faster than $2^{\circ} \mathrm{C} / \mathrm{hr}$ to avoid the ugly consequences of "thermal-shock" that could follow rapid neonatal temperature changes. ${ }^{1,9}$

\section{Validation and clinical trial}

The new algorithm was implemented at the SCBU of UATH Abuja after several theoretical and practical courses on ISA were carefully completed for interested members of SCBU staff team. Neonates weighing up to $1200 \mathrm{~g}$ at birth and those of gestational-age of 32 completed weeks or less were all recruited into the study based on UATH Ethical Approval No: FCT/UATH/HREC/PR/506. In order to be included in the test-patient group, informed consents were obtained from each mother after careful explanation of all the study was trying to achieve. Every question presented during the consent meetings was duly answered to the satisfaction of the mothers.

Neonatal body temperatures and other physiological vital signs were noted at the point-of-admission (POA) for every neonate presenting at the centre. All temperatures were taken via the axilla. Other procedures, including initial examinations and resuscitations as normally practiced at the centre were carried out. All other clinical and nursing techniques were left unaltered to ensure that the only parameter to differentiate the 'test' cases from the cohorts of our prospective and retrospective 'controls' was the manner of initial body temperature stabilisation. The expectant incubator was usually switched 'ON' up to 45 minutes before expected time of neonate's arrival. The WUSP value was chosen as described in section 2.1-1 and initial-setpoint (Ti) for the commencement of neonatal nursing was finally calculated upon newborn's arrival using the ISA as already described (section 2.1-2). The educated WUSP helps to eliminate the long periods of waiting for incubator to appropriately warm up for the neonate if this was turned 'ON' only upon neonate's arrival.

The ISA was deployed without delays whenever the need arose. Ti was calculated to re-initiate incubator care every time neonate was removed from the incubator for procedures outside the unit. All information of "time and body temperature" during vital signs checks were recorded for each of such cases. Neonates were also kept under this special monitoring for the rest of their first seven days of life. Specific temperature data for this study were extracted from the neonate's case file after the F7D period or after death if this happened before F7D. This includes:

\section{i. Point-of-admission (POA) time and temperature}

ii. Incubator WUSP temperature and the final initial setpoint.

iii. Neonate's body temperatures and times for commencing or recommencement of incubator care as indicated in the patients' treatment files.

iv. Times and body temperatures during subsequent re-assessments and vital signs checks - typically carried out every hour for the cohorts of our test cases. Exceptionally, for neonates presenting body temperatures outside the safe-zone, vital signs were documented every 15 to 30 minutes and incubator setpoint optimally reset using the handy-approach technique. ${ }^{8}$ This procedure was continued until the neonate was thermally stabilised. The neonate was defined to have thermally stabilised if patient's temperature remained within the safe zone for a minimum of 1 hour, without any episode of over- or under-shoot into hyperthermia or hypothermia regions, respectively.

\section{Data inclusions and exclusions}

The most vulnerable, high mortality and highest risk neonates to poor thermal control were adjudged the best candidates to test the effectiveness of the ISA. Hence, extremely low birth weight (ELBW, $<1000 \mathrm{~g}$ ) neonates and neonates at the lower margins of very low birth weight category $(1000 \mathrm{~g}-1200 \mathrm{~g})$ were recruited for study. The intensive training and trials for the application of the ISA was completed in May 2015. The "Test" cases were set to include neonates presenting after May 2015. The "Controls" were cases that could be considered as completely uninfluenced by the learning periods of the use of ISA. Additional exclusion criteria within the already indicated limits of birth parameter were as follows:

I. Neonates of gestation-age below 25 completed weeks.

II. Patients within the acceptable BW but with GA $>32$ completed weeks.

III. Patients with the acceptable GA but with BW equal or less than $550 \mathrm{~g}$.

IV. Patients presenting lifeless at POA

V. Patients presenting with positive history of ruptured membrane prior to delivery. 
Table I Morbidity summary

\begin{tabular}{|c|c|c|c|c|c|c|}
\hline \multirow{2}{*}{$\begin{array}{l}\text { Disease } \\
\text { Presenting }\end{array}$} & \multicolumn{3}{|c|}{ Control-Historic (62) } & \multicolumn{3}{|l|}{ Test (27) } \\
\hline & Patients & av.episode per patient & Rate $(/ 100)$ & Patients & av episode per patient & Rate $(/ 100)$ \\
\hline Apnoea & 30 & 2.1 & 48.4 & 4 & 1 & 14.8 \\
\hline RD & 55 & 1 & 88.7 & 20 & 1 & 74.1 \\
\hline Anaemia & 26 & 1.5 & 41.9 & 10 & 2 & 37 \\
\hline Malaria & 18 & I & 29 & 10 & 1 & 37 \\
\hline Sepsis & 26 & 1 & 41.9 & 6 & 1.5 & 22.2 \\
\hline НуроТ & 54 & 6.2 & 87.1 & 24 & 4 & 88.9 \\
\hline HyperT & 44 & 3.1 & 71 & 18 & 3.3 & 66.7 \\
\hline HyperB & 35 & I & 56.5 & 18 & 1 & 66.7 \\
\hline HypoG & 2 & 1 & 3.2 & 18 & 4.8 & 66.7 \\
\hline Acidosis & 28 & I & 45.2 & 10 & 1.4 & 37 \\
\hline Azotaemia & 2 & 1 & 3.2 & 2 & I & 7.4 \\
\hline HypoN & 12 & 1 & 19.4 & 1 & I & 3.7 \\
\hline HyperN & 3 & I & 4.8 & 0 & 0 & 0 \\
\hline HyperK & 5 & 1.2 & 8.1 & 4 & I & 14.8 \\
\hline НуроС & 4 & I & 6.5 & I & I & 3.7 \\
\hline HypoK & I & I & 1.6 & 0 & 0 & 0 \\
\hline
\end{tabular}

RD, respiratory distress; sepsis, anaemia, malaria, septicaemia; HyperB, hyperbilirubinemia; hypot, hypothermia; HyperT, hyperthermia; HypoG, hypoglycaemia; HypoN, hyponatraemia; HyperN, hypernatraemia; HyperK: hyperkalaemia; HypoK, hypokalaemia; HypoC, hypocalcaemia.

Table 2 p-values for inter-group similarities

\begin{tabular}{|c|c|c|}
\hline Significant Similarity p-value & Parameter & p -value \\
\hline & BW & 0.050 \\
\hline \multirow[t]{9}{*}{0.05} & GA & 0.010 \\
\hline & POA.temp & 0.002 \\
\hline & Resp.Distress & 0.146 \\
\hline & Anaemia & 0.049 \\
\hline & Malaria & 0.080 \\
\hline & Septicaemia & 0.197 \\
\hline & Hypothermia & 0.018 \\
\hline & Hyperthermia & 0.043 \\
\hline & Hyperbilirubinaemia & 0.102 \\
\hline \multirow[t]{8}{*}{0.1} & Hypoglycaemia & 0.634 \\
\hline & Acidosis & $0.08 \mathrm{I}$ \\
\hline & Azotaemia & 0.042 \\
\hline & Hyponatraemia & 0.157 \\
\hline & Hypernatraemia & 0.048 \\
\hline & Hyperkalaemia & 0.068 \\
\hline & Hypocalcaemia & 0.027 \\
\hline & Hypokalaemia & 0.016 \\
\hline
\end{tabular}

BW, birthweight; GA, gestational age; POA temp, point of admission temperature; Resp, respiratory

Table 3 Thermal stability summary

\begin{tabular}{|c|c|c|c|c|c|c|}
\hline \multirow[t]{2}{*}{ Parameter } & \multicolumn{3}{|c|}{ Control } & \multicolumn{3}{|l|}{ Test } \\
\hline & Mean & $95 \% \mathrm{Cl}$ & Range & Mean & $95 \% \mathrm{Cl}$ & Range \\
\hline BW (g) & 1013 & 26.6 & $600-1200$ & 1010 & 58.6 & $600-1200$ \\
\hline GA (completed weeks) & 29 & 0.3 & $27-32$ & 28 & 0.5 & $26-32$ \\
\hline POA.temp $\left({ }^{\circ} \mathrm{C}\right)$ & 36 & 0.2 & $34.5-38.9$ & 36.0 & 0.4 & $34.5-36.4$ \\
\hline Waiting for incubator (hrs) & 8.4 & 1.6 & $3.8-32.8$ & 1.5 & 0.6 & $0-5.9$ \\
\hline Initial stabilization (hrs) & 12.4 & 2.3 & $0.3-47.5$ & 0.47 & 0.2 & $0.1-2.0$ \\
\hline \multicolumn{7}{|c|}{ Exposures outside safe-zone temperatures (hours) } \\
\hline Cumulative/ patient & 65.1 & 6.0 & $3.6-128.8$ & 23.9 & 7.0 & $0-89.9$ \\
\hline Exposure per incidence & 12.1 & 1.5 & $3.6-58.8$ & 5.1 & 1.0 & $4.7-15$ \\
\hline Hypothermia per 100 nurs-hrs & 39.9 & 2.3 & $0-100$ & 9.6 & 4.7 & $0.7-44.5$ \\
\hline Hyperthermia per 100 nurs-hrs & 11.8 & 4.7 & $0-88.4$ & 4.7 & 3.5 & $0-23.7$ \\
\hline Hospital stay (days) & 29.5 & 3.3 & $11-52$ & 28.6 & 1.8 & $12.0-53.0$ \\
\hline
\end{tabular}

$\mathrm{Cl}$, confidence interval, BW, birthweight, GA, gestational age, POA. Temp, point of admission temperature, nurs-hrs - nursing hours

Citation: Amadi HO,Adesina CT, Olateju EK, et al.Validation of a novel technique that minimises early neonatal deaths -a comparative Study.J Pediatr Neonatal Care. 20I7;6(I):24-3I. DOI: 10.15406/jpnc.2017.06.00232 


\section{Control cohorts}

Comparative analyses could only be justified if incidences of morbidity had remained the same and if any practice changes other than the ISA had not significantly improved outcomes at the unit over the years. Hence it was necessary to examine three sets of previous and contemporary cases to verify any justification for the comparison. Previously managed patients with requisite information within the gestational age and birth weight being investigated were identified from the unit's Patient-register. The relevant case files were recalled from the hospital's Records Department. These were thoroughly assessed and the relevant temperature data and final outcomes extracted. The three sets of control data were:

a. Historic cases: Involving all available and qualifying cases between 2009 and 2011. This would be related to the outcomes of previously published studies by Okechukwu and Achonwa from 2005-2006 data; ${ }^{3}$ and Onalo and Olateju from 2006-2010 data. ${ }^{4}$

b. Recent past cases: Involving all qualifying data from January 2014 to April 2015 when training for ISA began.

c. Contemporary cases: Involving all qualifying prospective candidates between June 2015 and June 2016 that were not recruited into the Test patient group but managed conventionally without the ISA application. Qualifying candidates were excluded from test-group if (a) neonate's mother refused to offer her consent for inclusion in the new ISA application or (b) there was no trained research group member to initiate the ISA procedures during the working shift of neonate's arrival.

These groups of cases were applied as 'control data' for a comparative analysis against the test- cases that were managed with ISA technique between June 2015 and June 2016. Overall F7D and ultimate mortality rates of all categories were quantified in terms of number of deaths per 100 admissions and compared to evaluate how the ISA application might have affected the unit's outcomes.

\section{Analysis}

Each neonate's vital signs of temperature were extracted from the patient files for up to 8 days from the commencement of incubator care following admission. Microsoft Excel software was applied for the analysis of the extracted data. All cases were individually plotted in Microsoft excel graphs of body temperature against lifetime duration (Figure 3), covering the first 168 hours of life. Points of commencement or re-commencement of incubator care were identified and indicated on the charts too. This clearly revealed any stabilisation achieved and how long it took to achieve this. These were extracted for further processing. Neonatal morbidities presenting during the F7D period were extracted and separately tabulated to investigate how thermal instability might perhaps be associating with these. The 'Control' and 'Test' patient categories were each separated into three sub-groups based on birth weight, namely: $<801 \mathrm{~g}, 801-1000 \mathrm{~g}$ and 1001-1200 g to investigate and compare mortality rates of the subgroups.

\section{Justifications for comparison}

The incidences of co-morbid factors other than initial thermal distress were examined to confirm that these factors were equally incident in both Test and historic-Control patient groups. All presenting co-morbid factors and their total number of reoccurrence for each patient were extracted from the files. These were among others: respiratory distress (RD), anemia, malaria, septicemia (Sepsis), hyperbilirubinemia (HyperB), hypothermia (HypoT), hyperthermia (HyperT), hypoglycemia (HypoG) and acidosis. The patient morbidity rate was quantified in terms of the number of patients presenting with these conditions out of every 100 neonates. Null hypotheses for significant similarity in incidence rate for these conditions between the Control and Test groups was set at level $(\mathrm{p}=0.1)$. This was calculated based on the differences between the values of the corresponding morbidity rates in the test and control groups. Other than co- morbid factors, similarities of other fundamental inter-group factors that might tilt the balance for comparison - such as birth weight, gestationage and POA-temperature were assessed. Significant similarity level was set at $(\mathrm{p}=0.05)$. All or most of the 18 enlisted factors should be significantly present and similar between the two groups to justify comparative investigation.

\section{Quantified parameters}

The mean and $95 \%$ confidence-interval $(95 \% \mathrm{CI})$ of various parameters were quantified. These included:

a. The time lapse before the thermal stabilisation of each category of neonates.

b. The waiting time before the commencement of incubator care.

c. Thermal-stress morbidity rates defined in terms of hypothermia or hyperthermia hours per 100 hours of nursing.

d. Neonatal co-morbidity rate defined as the number of neonates presenting with a particular co-morbid factor per 100 patients.

e. The F7D mortality rate defined as the fraction of neonatal deaths occurring within the first seven days of life - quantified in terms of number of neonates that could not live beyond F7D in every presenting 100 .

f. The consequential overall neonatal mortality rate (NNMR) defined as the number of patients that eventually died before successful discharge in every 100 cases.

g. The fraction of deceased neonates that died within the F7D period (f7d-in-NNM).

\section{Result}

A total of 105 cases formed the Control-patient group comprising of 64 historic (2009-2011) and 41 recent (2014-2016) cases. The mean

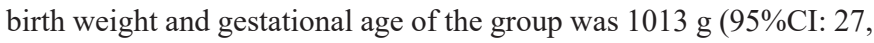
range: $600-1200)$ and 29 completed weeks (95\%CI: 0.3, range: $27-$ 32), respectively. There were 29 cases in the Test-patient group; mean

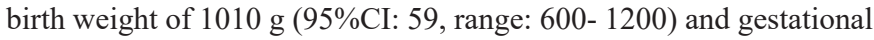
age of 28 completed weeks (95\%CI: 0.5, range: 26-32). Both test and historic-control patient groups presented with incidences of the enlisted co-morbid factors with 78\% (14 out of 18) proportional similarities in the distribution of co-morbid factors in both groups (Tables $1 \& 2$ ). Neonatal apnoea was significantly dissimilar (Figure 4). The mean waiting time before commencement of incubator care was $1.5 \mathrm{hrs}(95 \% \mathrm{CI}: 0.6)$ in the Test group and $8.4 \mathrm{hrs}(95 \% \mathrm{CI}: 1.6)$ for the Control-group. On the average, it took 28 minutes to achieve the initial normotherm in the Test-group as compared to 12 hours and 24 minutes for the Control-group. Other comparative details, including resultant accumulated length of exposure to hypothermia and hyperthermia per 100 hours of nursing were presented in Table 3. On the overall, the resultant neonatal mortality rate for the Test-group was less than $7 \%$ (2 out of 29 ) as compared to $50 \%$ (32 of 64) in the Historic-cases group and 63\% (26 of 41) in the recent-cases group. More than half of the deceased patients in the Control died within their F7D period - 20 of 32 in the historic-cases and 21 of 26 in the recent-cases. There was no F7D mortality in the test- group. The most 
vulnerable sub-group was $600 \mathrm{~g}-800 \mathrm{~g}$ birth weight. Virtually all of these (18 out of 19) in the control-groups were lost before the end of their F7D period. All four cases in the Test-group survived (Figure 5).

\section{Discussion}

Earlier studies involving quantification of mortality rates at the Special Care Baby Unit (SCBU) of University of Abuja Teaching Hospital (UATH) clearly revealed excessively high newborn deaths., ${ }^{3,4}$ This is consistent with the conditions in similar Nigerian tertiary hospitals. ${ }^{10,12}$ However, no Nigerian research publication has been able to synthesize any solutions for the reduction of the mortalities; ${ }^{13}$ hence prompting the introduction of the new techniques that led to the present investigation. Okechukwu and Achonwa (2009) studied mortality pattern at the SCBU of UATH, covering data between 2005 and 2006. This revealed that LBW and prematurity were among the greatest reasons for admission and death at the unit. A follow up study covering data from 2006 to 2010 was undertaken by Onalo and Olateju (2015) but this did not reveal any significant changes or improvement in the survival rate of neonates despite the recommendations of the earlier study from the unit. The highest mortality rates of extremely LBW (750/1000) and very LBW (643/1000) as quantified from 2006 data were similar to those for 2010 data, being $(732 / 1000)$ and $(664 / 1000)$, respectively $[3,4]$. Our control data for this present study - covering a more recent past cases, from January 2014 to June 2016 - shows that the mortality rate for ELBW (represented by $100 \%$ for $600-800 \mathrm{~g} \mathrm{BW}$ and $66.7 \%$ for $801-1000 \mathrm{~g} \mathrm{BW}$ ) is similar to the results of these past studies and has remained as high over the last 10 years (Figure 5). These figures were worse elsewhere at another Nigerian centre with NNMR for $<1000 \mathrm{~g}$ as high as $93.3 \%$ [14]. This clearly suggests an obvious absence of effective solution to improve the survival rate of premature neonates in the last 10 years. This is also representative of the entire Nigerian situation and may explain why the country could not achieve the millennium development goal target for the neonate.

The literature has identified various factors that exacerbated the neonatal risk of thermal stress in Nigeria, especially in the very- and extremely-LBW neonates. ${ }^{15-17}$ Extreme lack of adequate knowledge of neonatal thermal control was identified in these centres. This partly explained why the high mortality rates of these categories of neonates remained very high till date. ${ }^{1,6,8}$ The present analyses show that the relative number of neonates presenting with hypothermia or hyperthermia as signs of their response to changes in their immediate environmental temperatures was significantly similar $(\mathrm{p}=0.01$ and 0.06 , respectively) in both groups of patients. However, there were significant differences in the final outcome of the two groups (Figure 6 ). The average time taken to stabilize a newly arrived neonate using the ISA was quicker (28 minutes) in the Test-group, down from 12 hours in the Control-group. The supportive role ISA played ensured that the prolonged thermal-distress that might have led to a mortality of $71.2 \%$ in the first 72 hours of life as reported by an earlier study from the centre was eliminated. ${ }^{3}$ Hence, all the neonates in the present Test-group (29 of 29) survived their first 72 hours of life. Beyond this point, neonate's relative rate of exposure to hypothermia also reduced by more than half, from 40 hours per 100 nursing hours to 16 per 100. This also resulted in the huge difference in the overall neonatal mortality rate for the two groups as all the 29 Test-patients survived the F7D period; only two were lost but these happened much later than their F7D periods. However, the overall mortality rate for the Controlpatient groups remained as high as 63\% (Figure 7). The proportion of deceased neonates dying within the F7D period (f7d-in-NNM) remained between $63 \%$ and $81 \%$ for all the control-patient groups as presented in Figure 7. This is quite similar to the $79 \%$ reported by the WHO for Nigeria. ${ }^{7}$ Our present study has, perhaps coincidentally, completely reversed this as this figure has fallen to $0 \%$.

We think that the immediate postnatal long hours of traumatic thermal shock inflicted on the Control patients - as they remained outside the thermal safe-zone temperatures must have resulted in some lasting damage that trailed their subsequent sluggish recovery from other presenting co-morbidities. The effect of improved thermal stability due to ISA might have also reflected in the lower record of apnoea in the Test-group (Figure 4). The Test data revealed higher association with hypoglycemia (64/100 patients) as compared to $3 / 100$ for the historical-Control. We do not fully understand the reason for this trend other than the SCBU's present ability in quicker diagnosis with readily available handheld devices. Septicemia appeared significantly higher in the historical-Control. This might, in part, be due to intensified use of hand sanitizing gel and improved water supply to the unit as the unit did not introduce any other different septic measures during the two comparing periods of time. The contents of the historic-control data also suggest the possibility of wrongly diagnosing 'disease processes' as the reason for some periodic hyperthermia that we can now blame on neonatal exposure to uncontrollable high environmental temperatures. ${ }^{17}$

The ISA is therefore recommended for neonatal units that must lower NNMR in a resource-constrained setting.

\section{Study limitations}

A number of situations posed some difficulties in this investigative study. These include:

I. The short duration of the study leading to fewer test-cases as compared to the control sample size.

II. Frequent inter- and intra-departmental reshuffling of health personnel as occasioned by the hospital's personnel management. Some of these often affected nurses and resident doctors that had been trained on the techniques thereby limiting prompt recruitment of some qualifying cases into the test sample population.

III. Some of the neonates arriving by referral from other places might have stayed a long period out of the thermal safe zone prior to presentation and subsequent application of the ISA.

IV. There were also incessant hospital workers' strike during the period of the study; this limited the test sample size and hampered best delivery of services.

\section{Acknowledgements}

We wish to thank all UATH SCBU staff members for their hard work during the entire duration of this difficult centre control study. This research was funded in part by Neonatal Concerns for Africa and Polchazum Engineering Limited, subsidiaries of Mastermind group, Nigeria. We also acknowledge Virgin Atlantic Airways, United Kingdom and Hornchurch Baptist Church, United Kingdom who partly supported Professor H Amadi's medical missions in Nigeria leading to this publication.

\section{Conflict of interest}

The authors declare no conflict of interest.

\section{Funding}

None. 


\section{References}

1. Amadi HO, Olateju EK, Kawuwa MB, et al. Neonatal hyperthermia and thermal stress in low- and middle-income countries:a hidden cause of death in extremely low-birth weight neonates. Paediatr Int Child Health. 2015;35(3):273-281.

2. Mullany LC. Neonatal hypothermia in low-resource settings. Semin Perinatol. 2010;34(6):426-433.

3. Okechukwu AA, Achonwa A. Morbidity and mortality patterns of admissions into the special care baby unit of University of Abuja Teaching Hospital, Gwagwalada, Nigeria. Niger J Clin Pract. 2009;12(4):389-394.

4. Onalo R, Olateju EK. A four-year retrospective review of very low birth weight babies seen at the University of Abuja Teaching Hospital Nigeria. Nig Postgrad Med J. 2015;22(2):93-99.

5. Onyiriuka AN. Trend in the incidence of delivery of low birth weight infants in Benin City, Southern Nigeria. Nig Postgrad Med J. 2006;13(3):189-194.

6. Amadi $\mathrm{HO}$, Osibogun $\mathrm{AO}$, Eyinade $\mathrm{O}$, et al. Challenges and frugal remedies for lowering facility based neonatal mortality-a comparative study. Inter J Pediatr. 2014;2014:986716.

7. WHO African Region. Nigeria:Maternal and perinatal health profile. Department of Maternal, Newborn, Child and Adolescent Health. World Health Organisation. 2013.

8. Amadi HO. Neonatal thermo neutrality in a tropical climate. In:Rodriguez-Morales AJ, editor. Current Topics in Tropical Medicine. In Tech, Rijeka 51000, Croatia. 2012. p. 513-544.
9. Konopova P, Janota J, Termerova J, et al. Successful treatment of profound hypothermia of the newborn. Acta Paediatr. 2009;98(1):190 192.

10. Ogunlesi TA, Ogunfowora OB, Adekanmbi A, et al. Point-ofadmission hypothermia among high-risk Nigerian newborns. $B M C$ Pediatr. 2008;8:40.

11. Federal Ministry of Health Nigeria. Saving newborn lives in Nigeria:Newborn health in the context of the Integrated Maternal, Newborn and Child Health Strategy. $2^{\text {nd }}$ ed. Abuja. Federal Ministry of Health, Save the Children, Jhpiego. 2011.

12. You D, Rou-New J, Wardlaw T. Levels and trends in child mortality -report 2012. UNICEF, USA;2012.

13. Kana MA, Doctor HV, Peleteiro B, et al. Maternal and child health interventions in Nigeria:a systematic review of published studies from 1990 to 2014. BMC Pub Health. 2015;15:334.

14. Ogunlesi TA. Factors influencing the survival of newborn babies weighing $<1.5 \mathrm{~kg}$ in Sagamu, Nigeria. Arch Gynecol Obstet. 2011;284(6):1351-1357.

15. Amadi HO, Mokuolu OA, Adimora GN, et al. Digitally recycled incubators:better economic alternatives to modern systems in lowincome countries. Ann Trop Paediatr. 2007;27(3):207-214.

16. Amadi HO, Azubuike JC, Etawo US, et al. The impact of recycled neonatal incubators in Nigeria:a 6-year follow-up study. Int J Pediatr. 2010;2010:269293.

17. Amadi HO, Mohammed IL, Kawuwa MB, et al. Synthesis and validation of a weatherproof nursery design that eliminates tropical EveningFever-Syndrome in neonates. Int J Pediatr. 2014:9. 